\title{
法政大学学術機関リポジトリ
}

Perfor mance Eval uat i on of Several I mpl i ci t FDTD Met hods for Opt i cal Wavegui de Anal yses

\begin{tabular}{|l|l|}
\hline 著者 & $\begin{array}{l}\text { SH BAYANA Jun, MRAK M t sunor i, TAKAHASH } \\
\text { Ryo, YAMAUCH J unj i , NAKANO H samat su }\end{array}$ \\
\hline 出版者 & I EEE \\
\hline $\begin{array}{l}\text { j our nal or } \\
\text { publ i cat i on t i t l e }\end{array}$ & Jour nal of I i ght wave t echnol ogy \\
\hline number & 6 \\
\hline page r ange & $2465-2472$ \\
\hline year & $2006-06$ \\
\hline URL & ht t p: //hdl . handl e. net /10114/233 \\
\hline
\end{tabular}




\section{Performance Evaluation of Several Implicit FDTD Methods for Optical Waveguide Analyses}

Jun Shibayama, Member, IEEE, Member, OSA, Mitsunori Muraki, Ryo Takahashi, Junji Yamauchi, Member, IEEE, Member, OSA, and Hisamatsu Nakano, Fellow, IEEE

\begin{abstract}
The performance of the recently developed implicit finite-difference time-domain methods (FDTDs) is compared with that of the traditional explicit FDTD. For the implicit methods, the alternating-direction implicit (ADI) FDTD and the envelope ADI-FDTD are investigated. In addition, the locally one-dimensional (LOD) scheme is newly introduced into implicit FDTDs, i.e., LOD- and envelope LOD-FDTDs are developed for simple implementation of the algorithm and reduced CPU time. Numerical dispersion analysis is performed, demonstrating the improved dispersion properties of the envelope FDTD. Numerical results of a waveguide grating reveal that the wavelength responses obtained from the ADI/LOD-FDTD gradually shift toward a longer wavelength as the time step $(\Delta t)$ is increased. For the envelope ADI/LOD-FDTD with $\Delta t=8 \Delta t_{\mathrm{CFL}}$, in which $\Delta t_{\mathrm{CFL}}$ is determined by the stability criterion, the responses are in good agreement with the response of the explicit FDTD, showing the comparable CPU time to that of the explicit FDTD. Further calculations of a waveguide with high-reflection coatings reveal that the CPU time of the envelope LOD-FDTD with $\Delta t=32 \Delta t_{\mathrm{CFL}}$ is reduced to $25 \%$ of that of the explicit FDTD.
\end{abstract}

Index Terms-Alternating-direction-implicit (ADI) scheme, beam-propagation method (BPM), finite-difference time-domain method (FDTD), locally one-dimensional (LOD) scheme, numerical dispersion, optical waveguide, waveguide grating.

\section{INTRODUCTION}

$\mathbf{T}$ HE FINITE-DIFFERENCE time-domain method (FDTD) [1], [2] has widely been used to obtain wideband characteristics of optical waveguides and devices [3]-[5]. Note, however, that the Courant-Friedrich-Levy (CFL) condition prevents us from using a large time step $(\Delta t)$. On the other hand, the TD beam-propagation method (TD-BPM) [6] has been developed, in which only the slowly varying envelope (SVE) is analyzed, considering the fact that the modulation frequency is much lower than the optical carrier frequency. Since the TD-BPM is generally based on the implicit scheme, CFL condition does not exist, allowing us to use a large $\Delta t$. We have compared the performance of the explicit FDTD and the TD-BPM for the analyses of optical waveguides [7].

Recently, the CFL condition of the explicit FDTD has been removed [8]-[11] with the alternating-direction-implicit (ADI) scheme [12], [13]. The ADI-FDTD has been assessed from

Manuscript received October 28, 2005; revised February 26, 2006. This work was supported in part by the "University-Industry Joint Research" Project for Private Universities: matching fund subsidy from Ministry of Education, Culture, Sports, Science and Technology (MEXT), 2003-2007.

The authors are with the Faculty of Engineering, Hosei University, Tokyo 184-8584, Japan (e-mail: shiba@k.hosei.ac.jp; j.yma@k.hosei.ac.jp; nakano@k.hosei.ac.jp).

Digital Object Identifier 10.1109/JLT.2006.874570 various aspects in the microwave community [14]-[18]. In addition, to reduce the numerical dispersion error, the envelope ADI-FDTD has been developed [19] on the basis of the same concept as that of the TD-BPM. We have also proposed an efficient implicit FDTD based on the locally one-dimensional (LOD) scheme [20]. The LOD-FDTD reduces the CPU time while maintaining the accuracy comparable to the ADI-FDTD. Although several implicit FDTDs have been studied, as discussed above, a comparison among these methods has not yet been fully made, particularly for the optical waveguide analyses.

This paper is the sequel to our previous publication [7] and investigates the performance of implicit FDTDs, such as the ADI-, LOD-FDTDs, and their envelope versions, in comparison with the traditional explicit FDTD.

We first review the formulations of the implicit FDTDs and newly develop an envelope LOD-FDTD. After presenting the formulation, we perform the numerical dispersion analysis, paying attention to the choice of $\Delta t$. It is shown that for the ADI/LOD-FDTD, the normalized phase velocity gradually degrades with an increase in $\Delta t$, while for the envelope ADI/LOD-FDTD, the dispersion property is improved, in which the phase velocity always remains close to unity at the center wavelength. We next assess the computational accuracy and efficiency through analyzing the spectral response of the reflection coefficient for a waveguide grating [7]. Numerical results show that the responses obtained from the ADI/LODFDTD gradually shift towards a longer wavelength, as $\Delta t$ is increased. In contrast, the responses from the envelope $\mathrm{ADI} / \mathrm{LOD}-\mathrm{FDTD}$ with $\Delta t=8 \Delta t_{\mathrm{CFL}}$, where $\Delta t_{\mathrm{CFL}}$ is determined by the CFL condition, are in good agreement with the response of the explicit FDTD ranging from 1.2 to $2.0 \mu \mathrm{m}$, while offering the comparable CPU time to that of the explicit FDTD. Further calculations of a waveguide with high-reflection coatings reveal that the CPU times of the envelope LOD-FDTD are reduced to $50 \%$ and $25 \%$ for $\Delta t=16 \Delta t_{\mathrm{CFL}}$ and $32 \Delta t_{\mathrm{CFL}}$, respectively, of those of the explicit FDTD.

\section{TD METHODS}

\section{A. Explicit FDTD Method}

We consider linear, isotropic, and lossless materials in a twodimensional problem. Maxwell's equations are expressed as

$$
\frac{\partial \tilde{\phi}}{\partial t}=([A]+[B]) \tilde{\phi}
$$


where $\tilde{\phi}=\left[\tilde{H}_{x}, \tilde{H}_{z}, \tilde{E}_{y}\right]^{\mathrm{T}}$, and

$$
[A]=\left[\begin{array}{ccc}
0 & 0 & \frac{\partial}{\mu_{0} \partial z} \\
0 & 0 & 0 \\
\frac{\partial}{\epsilon_{0} n^{2} \partial z} & 0 & 0
\end{array}\right] \quad[B]=\left[\begin{array}{ccc}
0 & 0 & 0 \\
0 & 0 & -\frac{\partial}{\mu_{0} \partial x} \\
0 & -\frac{\partial}{\epsilon_{0} n^{2} \partial x} & 0
\end{array}\right]
$$

for the TE mode, and $\tilde{\phi}=\left[\tilde{E}_{x}, \tilde{E}_{z}, \tilde{H}_{y}\right]^{\mathrm{T}}$ and

$$
[A]=\left[\begin{array}{ccc}
0 & 0 & -\frac{\partial}{\epsilon_{0} n^{2} \partial z} \\
0 & 0 & 0 \\
-\frac{\partial}{\mu_{0} \partial z} & 0 & 0
\end{array}\right] \quad[B]=\left[\begin{array}{ccc}
0 & 0 & 0 \\
0 & 0 & \frac{\partial}{\epsilon_{0} n^{2} \partial x} \\
0 & \frac{\partial}{\mu_{0} \partial x} & 0
\end{array}\right]
$$

for the TM mode, in which $\epsilon_{0}$ and $\mu_{0}$ represent permittivity and permeability of a vacuum, respectively. $n$ is the refractive index of the waveguide. For the explicit FDTD, (1) is solved by the leapfrog algorithm. The upper limit of the time step is determined by the CFL condition.

\section{B. Implicit FDTD Methods}

The application of the implicit scheme to the discretization of (1) can eliminate the CFL condition. Applying the Crank-Nicolson (CN) scheme to (1) gives

$$
\tilde{\phi}^{l+1}=\frac{[I]+\frac{\Delta t}{2}([A]+[B])}{[I]-\frac{\Delta t}{2}([A]+[B])} \tilde{\phi}^{l}
$$

where $I$ denotes the unit matrix. Factoring (2) results in

$$
\tilde{\phi}^{l+1}=\frac{\left([I]+\frac{\Delta t}{2}[A]\right)\left([I]+\frac{\Delta t}{2}[B]\right)}{\left([I]-\frac{\Delta t}{2}[A]\right)\left([I]-\frac{\Delta t}{2}[B]\right)} \tilde{\phi}^{l} .
$$

A comparison between (2) and (3) reveals the addition of splitting error term in (3), i.e., $\Delta t^{2}[A][B] / 4$.

Equation (3) is successfully solved in two steps using the ADI scheme. As an alternative to the ADI scheme, we here apply the LOD scheme [13] to (3), so that

$$
\tilde{\phi}^{l+1 / 2}=\frac{[I]+\frac{\Delta t}{2}[B]}{[I]-\frac{\Delta t}{2}[B]} \tilde{\phi}^{l}
$$

for the first step and

$$
\tilde{\phi}^{l+1}=\frac{[I]+\frac{\Delta t}{2}[A]}{[I]-\frac{\Delta t}{2}[A]} \tilde{\phi}^{l+1 / 2}
$$

for the second step. From the first step (4a), we derive the following equations (although only the formulation for the TE case is discussed in the following, the TM case is similarly treated):

$$
\begin{aligned}
\tilde{H}_{x}^{l+1 / 2} & =\tilde{H}_{x}^{l} \\
\frac{\tilde{H}_{z}^{l+1 / 2}-\tilde{H}_{z}^{l}}{\Delta t / 2} & =-\frac{1}{\mu_{0}}\left(\frac{\partial \tilde{E}_{y}^{l+1 / 2}}{\partial x}+\frac{\partial \tilde{E}_{y}^{l}}{\partial x}\right) \\
\frac{\tilde{E}_{y}^{l+1 / 2}-\tilde{E}_{y}^{l}}{\Delta t / 2} & =-\frac{1}{\epsilon_{0} n^{2}}\left(\frac{\partial \tilde{H}_{z}^{l+1 / 2}}{\partial x}+\frac{\partial \tilde{H}_{z}^{l}}{\partial x}\right) .
\end{aligned}
$$

From the second step (4b), we have

$$
\begin{aligned}
\tilde{H}_{z}^{l+1} & =\tilde{H}_{z}^{l+1 / 2} \\
\frac{\tilde{H}_{x}^{l+1}-\tilde{H}_{x}^{l+1 / 2}}{\Delta t / 2} & =\frac{1}{\mu_{0}}\left(\frac{\partial \tilde{E}_{y}^{l+1}}{\partial z}+\frac{\partial \tilde{E}_{y}^{l+1 / 2}}{\partial z}\right) \\
\frac{\tilde{E}_{y}^{l+1}-\tilde{E}_{y}^{l+1 / 2}}{\Delta t / 2} & =\frac{1}{\epsilon_{0} n^{2}}\left(\frac{\partial \tilde{H}_{x}^{l+1}}{\partial z}+\frac{\partial \tilde{H}_{x}^{l+1 / 2}}{\partial z}\right) .
\end{aligned}
$$

Note that unlike the ADI-FDTD, in each half step of the LODFDTD, we move forward only in the $x$ - or $z$-direction. From (5) and (6), we finally derive the LOD-FDTD update equations for the TE mode. The unconditional stability of the LOD-FDTD is analytically confirmed in [20].

Recently, the envelope ADI-FDTD has been developed, in which only the SVE function is treated [19] as in the case of the TD-BPM. This envelope formulation can relax the constraint on the time step [21], [22]. Here, we extend this approach into the LOD-FDTD. Applying the time dependence $\tilde{\phi}=\phi e^{j \omega_{0} t}$, where $\phi=\left[H_{x}, H_{z}, E_{y}\right]^{\mathrm{T}}$ is the SVE function for the TE mode and $\omega_{0}$ is the center carrier frequency, to (1), we obtain

$$
\frac{\partial \phi}{\partial t}=\left([A]+[B]-j \omega_{0}[I]\right) \phi .
$$

We discretize (7) using the LOD scheme so that

$$
\begin{aligned}
H_{x}^{l+1 / 2} & =a_{-} / a_{+} H_{x}^{l} \\
\frac{a_{+} H_{z}^{l+1 / 2}-a_{-} H_{z}^{l}}{\Delta t / 2} & =-\frac{1}{\mu_{0}}\left(\frac{\partial E_{y}^{l+1 / 2}}{\partial x}+\frac{\partial E_{y}^{l}}{\partial x}\right) \\
\frac{a_{+} E_{y}^{l+1 / 2}-a_{-} E_{y}^{l}}{\Delta t / 2} & =-\frac{1}{\epsilon_{0} n^{2}}\left(\frac{\partial H_{z}^{l+1 / 2}}{\partial x}+\frac{\partial H_{z}^{l}}{\partial x}\right)
\end{aligned}
$$

for the first step, and

$$
\begin{aligned}
H_{z}^{l+1} & =a_{-} / a_{+} H_{z}^{l+1 / 2} \\
\frac{a_{+} H_{x}^{l+1}-a_{-} H_{x}^{l+1 / 2}}{\Delta t / 2} & =\frac{1}{\mu_{0}}\left(\frac{\partial E_{y}^{l+1}}{\partial z}+\frac{\partial E_{y}^{l+1 / 2}}{\partial z}\right) \\
\frac{a_{+} E_{y}^{l+1}-a_{-} E_{y}^{l+1 / 2}}{\Delta t / 2} & =\frac{1}{\epsilon_{0} n^{2}}\left(\frac{\partial H_{x}^{l+1}}{\partial z}+\frac{\partial H_{x}^{l+1 / 2}}{\partial z}\right)
\end{aligned}
$$

for the second step, where $a_{ \pm}=1 \pm j \omega_{0} \Delta t / 4$. We finally obtain two update equations in each step as

$$
\begin{aligned}
H_{z, i, j+1 / 2}^{l+1 / 2}= & a_{-} / a_{+} H_{z, i, j+1 / 2}^{l}-\frac{\Delta t}{2 a_{+} \mu_{0} \Delta x} \\
& \times\left(E_{y, i+1 / 2, j+1 / 2}^{l+1 / 2}-E_{y, i-1 / 2, j+1 / 2}^{l+1 / 2}\right. \\
& \left.\quad+E_{y, i+1 / 2, j+1 / 2}^{l}-E_{y, i-1 / 2, j+1 / 2}^{l}\right)
\end{aligned}
$$

$$
\begin{aligned}
E_{y, i+1 / 2, j+1 / 2}^{l+1 / 2}= & a_{-} / a_{+} E_{y, i+1 / 2, j+1 / 2}^{l}-\frac{\Delta t}{2 a_{+} \epsilon_{0} n^{2} \Delta x} \\
\times & \left(H_{z, i+1, j+1 / 2}^{l+1 / 2}-H_{z, i, j+1 / 2}^{l+1 / 2}\right. \\
& \left.\quad+H_{z, i+1, j+1 / 2}^{l}-H_{z, i, j+1 / 2}^{l}\right)
\end{aligned}
$$


for the first step, and

$$
\begin{aligned}
H_{x, i+1 / 2, j}^{l+1}= & a_{-} / a_{+} H_{x, i+1 / 2, j}^{l+1 / 2}+\frac{\Delta t}{2 a_{+} \mu_{0} \Delta z} \\
& \times\left(E_{y, i+1 / 2, j+1 / 2}^{l+1}-E_{y, i+1 / 2, j-1 / 2}^{l+1}\right. \\
& \left.\quad+E_{y, i+1 / 2, j+1 / 2}^{l+1 / 2}-E_{y, i+1 / 2, j-1 / 2}^{l+1 / 2}\right)
\end{aligned}
$$

$$
\begin{aligned}
E_{y, i+1 / 2, j+1 / 2}^{l+1}= & a_{-} / a_{+} E_{y, i+1 / 2, j+1 / 2}^{l+1 / 2}+\frac{\Delta t}{2 a_{+} \epsilon_{0} n^{2} \Delta z} \\
& \times\left(H_{x, i+1 / 2, j+1}^{l+1}-H_{x, i+1 / 2, j}^{l+1}\right. \\
& \left.\quad+H_{x, i+1 / 2, j+1}^{l+1 / 2}-H_{x, i+1 / 2, j}^{l+1 / 2}\right)
\end{aligned}
$$

for the second step.

Since (10a) includes the unknown term $E_{y}^{l+1 / 2}$, we substitute (10b) into (10a) in order to eliminate $E_{y}^{l+1 / 2}$ and implicitly solve the resultant tridiagonal equation. Then, (10b) is explicitly solved. Equations (11a) and (11b) can similarly be treated as that done in the first step, in which $a_{-} / a_{+} H_{x}^{l}$ is used for $H_{x}^{l+1 / 2}$ in (11a) and (11b) due to (8a).

It is worth mentioning that for the envelope LOD-FDTD, only two equations are solved in the half step. This contrasts with the case of the envelope ADI-FDTD, in which three equations should be solved. The present formulation significantly reduces the number of arithmetic operations, as in the case of the LOD-FDTD [20], with a subsequent reduction in the CPU time. It is clear that the equations of the envelope LOD-FDTD reduce to those of the LOD-FDTD for $\omega_{0}=0$.

It should be pointed out that the LOD formulations yield an additional error term whose coefficient is $\Delta t^{2}([A][B]-$ $[B][A]) / 4$. If $[A]$ and $[B]$ commute, the error term can be eliminated, leading to the second-order accuracy in time for the LOD formulations, as in the case of the ADI counterparts. Strictly speaking, the present LOD formulations are first-order accurate in time, due to the absence of commutativity of $[A]$ and $[B]$. Nevertheless, we have preliminarily confirmed that the numerical results obtained from the LOD-based FDTDs are in perfect agreement with those from the ADI-based FDTDs for all cases performed in Section IV. This is probably because the value of $([A][B]-[B][A])$ is negligibly small, and the subsequent additional error hardly affects the numerical results. Therefore, we assume that the ADI- and LOD-based methods are basically identical and denote the ADI- and LOD-FDTDs as implicit FDTD and their envelope versions as envelope FDTD (unless otherwise specified) in what follows.

\section{Other Related Methods}

Before leaving this section, we refer to some other methods related to the ADI-FDTD.

For the ADI-FDTD, the anisotropy of the numerical dispersion with respect to the wave-propagation angle increases with an increase in the time step. To compensate the anisotropy, the artificial permittivity has been introduced [23]-[25]. As an alternative, higher order FD methods have been employed to reduce the numerical dispersion error [26]-[31]. The higher order methods are also desirable for increasing the computational efficiency, since a large sampling width can be used in maintaining the accuracy of the numerical results. It should be noted, however, that a higher order ADI-FDTD has not yet been developed considering the boundary condition at a dielectric interface, which is indispensable for the optical waveguide analyses [7].

As discussed just below (3), the use of the ADI gives rise to the additional splitting error term. To reduce the error, an iteration procedure has been introduced in [32]. In addition, excitation schemes [33], [34] and perfectly matched layer (PML) boundary conditions [35]-[37] have been developed for the ADI-FDTD.

Although the methods mentioned above are not investigated in this paper, their applications to the optical waveguide analyses are interesting subjects of inquiry and are left for future study.

\section{NUMERICAL DISPERSION ANALYSIS}

It is important to study the wavelength characteristics of the numerical dispersion, since they offer useful information on the accuracy of the method by which the wideband characteristics are treated. The results also provide guidelines on the choice of $\Delta t$ for the FDTDs based on the implicit scheme. We here investigate the dispersion characteristics, paying attention to the choice of $\Delta t$.

The numerical dispersion relation is obtained by substituting a plane wave as

$$
e^{j\left(\omega p \Delta t-k_{x} q \Delta x-k_{z} m \Delta z\right)}
$$

where $p, q$, and $m$ denote the indexes for $t, x$, and $z$ axes, respectively, into the FD equations.

For the explicit FDTD, the numerical dispersion relation is expressed as [2]

$$
\sin ^{2}\left(\frac{\omega \Delta t}{2}\right)=r_{x}^{2}+r_{z}^{2}
$$

where $r_{\nu}=c \Delta t /(n \Delta \nu) \sin \left(k_{\nu} \Delta \nu / 2\right)$, in which $c$ is the speed of light in a vacuum, $k_{\nu}$ is the wavenumber, and $\nu=x$ or $z$.

The numerical dispersion relation for the implicit FDTD is obtained with the typical ADI case [16], [17] as

$$
\tan ^{2}\left(\frac{\omega \Delta t}{2}\right)=r_{x}^{2}+r_{z}^{2}+r_{x}^{2} r_{z}^{2}
$$

The numerical dispersion relation for the envelope version can be derived as

$$
\alpha^{3}=\beta^{2}\left(\alpha r_{x}^{2}+\alpha r_{z}^{2}+\tau_{-} r_{x}^{2} r_{z}^{2}\right)
$$


where

$$
\begin{aligned}
\alpha & =\frac{\omega_{0} \Delta t}{2} \tau_{+}+\left(1-\frac{\omega_{0}^{2} \Delta t^{2}}{16}\right) \tau_{-} \\
\beta & =-\tau_{+}+\frac{\omega_{0} \Delta t}{4} \tau_{-} \\
\tau_{+} & =2 j \cos \frac{\omega \Delta t}{2} \\
\tau_{-} & =2 j \sin \frac{\omega \Delta t}{2} .
\end{aligned}
$$

Notice that (14) reduces to (13) for $\omega_{0}=0$.

Since forward and backward waves traveling in the $\pm z$ directions predominate the properties of the waveguide, we only discuss the one-dimensional case in the $z$-direction [7], i.e., $r_{x}=0$.

Fig. 1(a) shows the normalized phase velocities for the implicit FDTD, and Fig. 1(b) and (c) shows the velocities for the envelope FDTD, in which the ratio of $\Delta t / \Delta t_{\mathrm{CFL}}$ is defined as the CFL number (CFLN). For reference, the result of the explicit FDTD is also included. Note that a spectral range of $0.8<k_{0} / k_{z}<1.33$ in Fig. 1 corresponds to that of $1.2<\lambda$ $<2.0 \mu \mathrm{m}$ in Fig. 3 for the grating analysis in Section IV. The phase velocity is calculated from $\omega /\left(k_{z} c / n\right)$ for the explicit and implicit FDTDs, and from $\left(\omega+\omega_{0}\right) /\left(k_{z} c / n\right)$ for the envelope FDTD, where $k_{z}=2 \pi n / \lambda$. In this calculation, the refractive index $n$ is chosen to be $\simeq 1.21$, which is close to the effective index of the TE mode at $1.5 \mu \mathrm{m}$ for the slab waveguide (without gratings) to be treated in Fig. 2. $\Delta z$ is set to be $0.04 \mu \mathrm{m}$ in Fig. 1(a) and (b) and to be $0.004 \mu \mathrm{m}$ in Fig. 1(c). For the explicit FDTD, although $\Delta t$ is chosen to be a slightly smaller value than that determined by the CFL condition, it is designated as $\Delta t_{\mathrm{CFL}}: \Delta t_{\mathrm{CFL}}=0.089 \mathrm{fs}$ in Fig. 1(a) and (b), and $\Delta t_{\mathrm{CFL}}=0.012 \mathrm{fs}$ in Fig. 1(c). These numerical parameters are consistent with those adopted for the numerical analysis performed in Section IV.

It is seen that the phase velocity is almost flat and close to unity for the explicit FDTD. In Fig. 1(a), the phase velocity for the implicit FDTD gradually degrades, as CFLN is increased. As will be seen later, this degradation seriously affects the results of the wideband response for the waveguide grating.

In contrast to the implicit FDTD, the velocity for the envelope FDTD remains close to unity at the center wavelength even for a large CFLN, as can be seen in Fig. 1(b). This means that the accuracy at the center wavelength is maintained even when a large $\Delta t$ is used. This is the reason why the envelope FDTD can substantially reduce the CPU time when the continuous wave excitation with a specific wavelength is adopted [19]. The dispersion characteristics in Fig. 1(b) indicate that the accuracy is maintained around the center wavelength when the pulse excitation is employed, although the accurate range shrinks as $\Delta t$ is increased. It is interesting to note that the dispersion characteristics of the envelope FDTD are similar to those of the full-band TD-BPM [7], since their formulations are closely related to each other: Temporal derivatives are fully incorporated into the formulation, and only the SVE function is calculated.

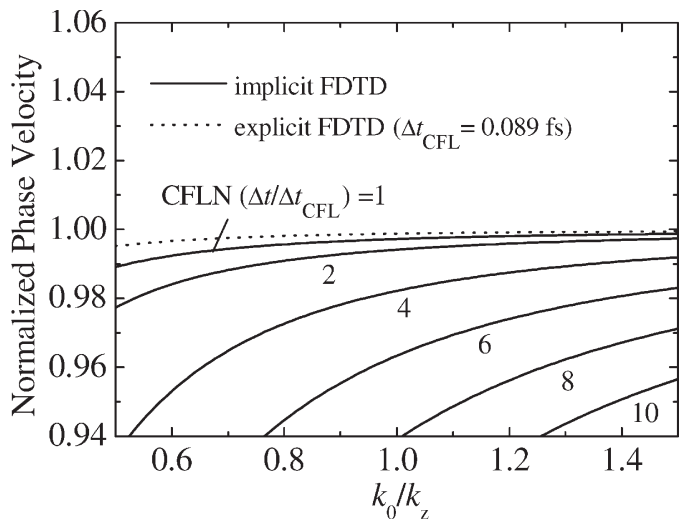

(a)

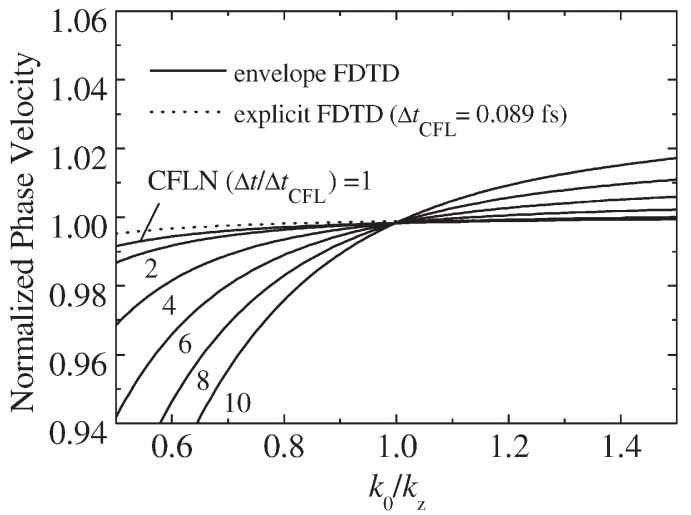

(b)

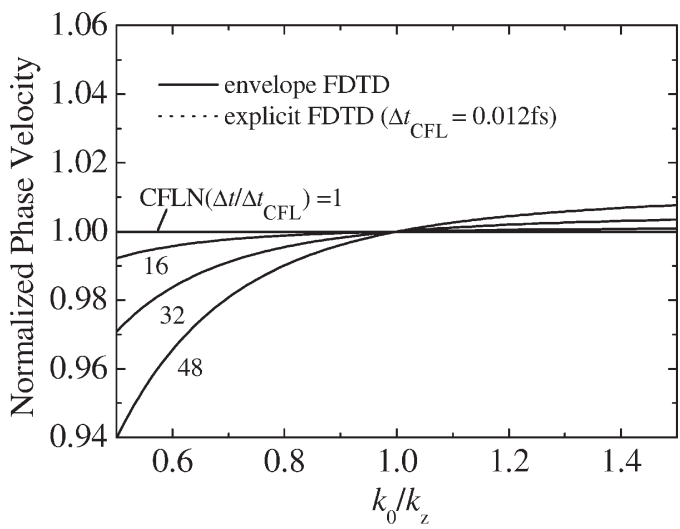

(c)

Fig. 1. Normalized phase velocity for (a) implicit FDTD with $\Delta z=$ $0.04 \mu \mathrm{m}$, (b) envelope FDTD with $\Delta z=0.04 \mu \mathrm{m}$, and (c) envelope FDTD with $\Delta z=0.004 \mu \mathrm{m}$. In (c), the result for CFLN $=1$ is superimposed on that of the explicit FDTD.

Fig. 1(c) shows the case for the envelope FDTD with a small spatial sampling width of $\Delta z=0.004 \mu \mathrm{m}$. This leads to the fact that $\Delta t_{\mathrm{CFL}}$ of the explicit FDTD also becomes small, resulting in a significant increase in the number of time steps in the practical simulation. On the contrary, for the envelope FDTD, there is no restriction on $\Delta t$, allowing the use of a large CFLN. In this case, the error of the phase velocity remains relatively low even for a large CFLN, as seen in Fig. 1(c). Therefore, the envelope FDTD is expected to efficiently provide the numerical results when small sampling widths are required for discretizing complicated structures with subsequent high accuracy. 


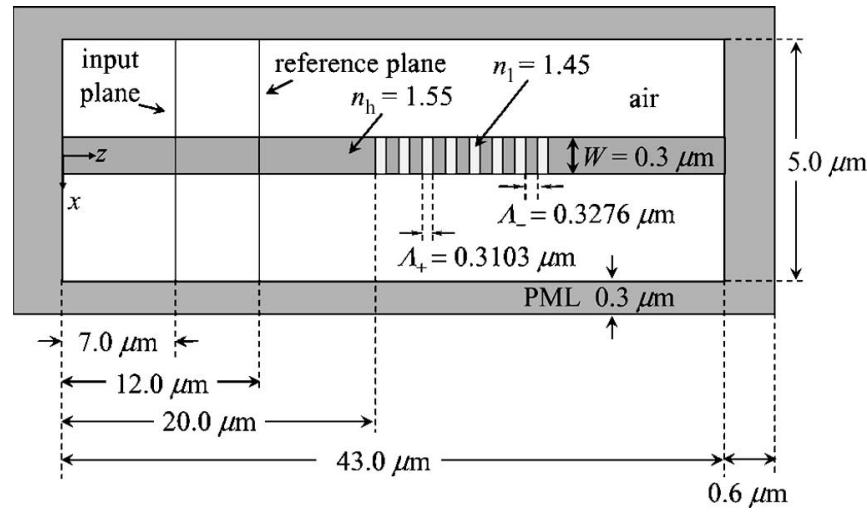

Fig. 2. Configuration of a waveguide grating.

In Section IV, we will examine how the error in the phase velocity affects the numerical results of the wideband response of the waveguide grating and the waveguide with high-reflection coatings.

\section{Numerical Results}

Fig. 2 illustrates the waveguide grating to be treated, in which the geometrical parameters are indicated. Due to the symmetry of the waveguide, only half of the section $(x>0)$ is analyzed. The pulse is excited at the input plane using the oneway excitation scheme [3], which consists of the eigenmode field in the $x$-direction and the Gaussian profile with a $1 / e$ full width of $4 \mu \mathrm{m}$ in the $z$-direction. We discretize the structure shown in Fig. 2 using the following spatial sampling widths: $\Delta x=3.75 \times 10^{-2} \mu \mathrm{m}$ and $\Delta z \simeq 3.99 \times 10^{-2} \mu \mathrm{m}$, in which the waveguide width and one grating period are divided by 8 and 16, respectively. These spatial sampling widths are the same as those used in [7]. For the explicit FDTD, the time step is set to be $\Delta t_{\mathrm{CFL}}=0.089 \mathrm{fs}$. These numerical parameters are consistent with those used in Fig. 1. The number of sampling points is $N_{x} \times N_{z}=74 \times 1110$, including eight and 16 points used for the PML in the $x$ - and $z$-directions, respectively. In this paper, the unsplit-type PML is used [38] for each method. Preliminary calculations show that the performance of the PML becomes worse, as $\Delta t$ of the implicit or envelope FDTD is increased. Fortunately, this does not seriously affect the accuracy of the numerical results in the following analysis.

The spectral response of the reflection coefficient is calculated from the ratio between the discrete Fourier transforms of the reflected pulse and the incident one observed at the reference plane illustrated in Fig. 2. The responses obtained from the implicit FDTD are shown in Fig. 3(a) (the results of the ADI-FDTD perfectly follow those of the LOD-FDTD). The response of the explicit FDTD with $\Delta t_{\mathrm{CFL}}$ is included for reference, which is indicated by the dotted line. Although not illustrated, the result of the implicit FDTD for CFLN $=1$ agrees well with that of the explicit FDTD. In Fig. 3(a), the results gradually degrade and shift rightwards, as CFLN is increased. This degradation may be expected from the results of the numerical dispersion analysis in Fig. 1(a). As will be seen later, the CPU time of the implicit FDTD becomes shorter than that of the explicit FDTD for CFLN $>4$. However, a large CFLN

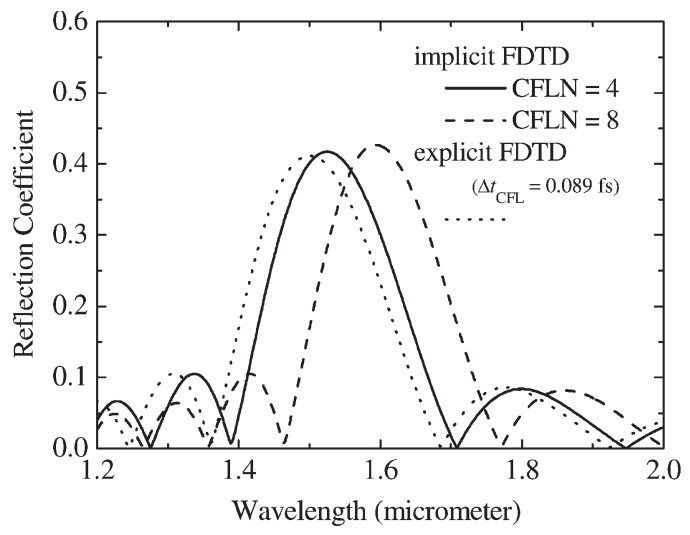

(a)

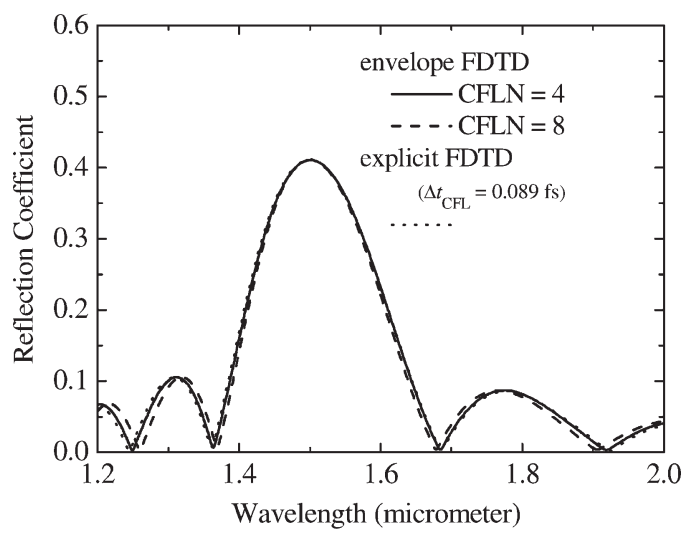

(b)

Fig. 3. Spectral response of the reflection coefficient. (a) Implicit FDTD and (b) envelope FDTD.

gives rise to the spectral-response shift, as shown in Fig. 3(a). Therefore, the implicit FDTD is not always advantageous to the wideband analysis of optical devices in terms of accuracy and efficiency, when compared with the explicit FDTD.

Fig. 3(b) depicts the spectral responses obtained from the envelope FDTD. The center wavelength is set to $1.5 \mu \mathrm{m}$. As expected from the results in Fig. 1(b), the responses agree well with those of the explicit FDTD around the center wavelength. Even for a large CFLN, reasonably accurate results are obtained over a wide spectral range. Careful observation reveals, however, that, at $\lambda<1.3 \mu \mathrm{m}$, the response for CFLN $=8$ slightly shifts rightwards. This wavelength range coincides with the range where the normalized phase-velocity error is larger than $1 \%$ in Fig. 1(b). As discussed in [7], in order to obtain an accurate result, the temporal and spatial sampling widths should be chosen so as to keep the dispersion error within $\pm 1 \%$.

In Fig. 4, the CPU time for each method is summarized for the TE-mode analysis, in which the total computational duration is $240 \mathrm{fs}$. We use a PC with a Pentium 4 extreme edition processor $(3.46 \mathrm{GHz})$. It is seen that the implicit and envelope FDTDs become efficient for CFLN $>4$ and CFLN $>8$, respectively, when compared with the explicit FDTD. The long CPU times of the envelope versions stem from the calculations of the complex values. It is noteworthy that the CPU times of the LOD-FDTD and its envelope version are reduced by $10 \%-15 \%$ when compared with the ADI counterparts, due to the simple algorithm of the LOD scheme. 


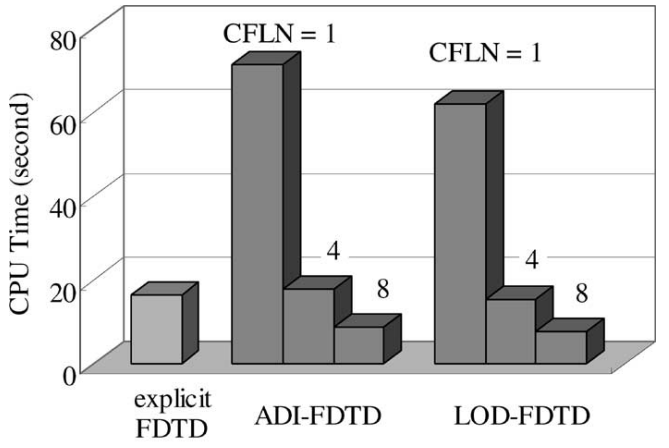

(a)

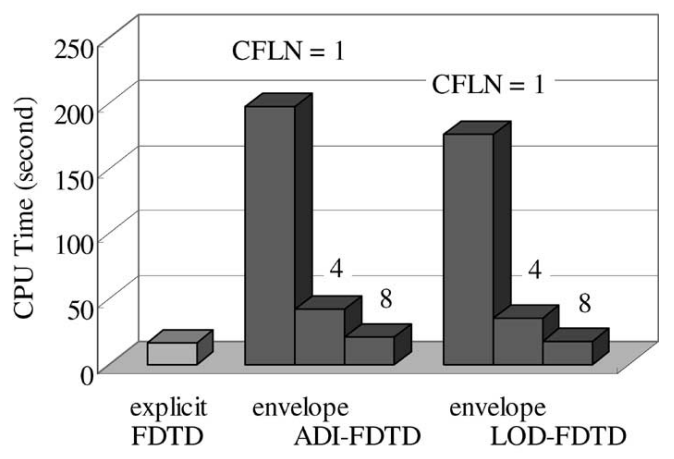

(b)

Fig. 4. CPU time. (a) Implicit FDTD and (b) envelope FDTD.

It should be noted that the efficiency of the implicit and envelope FDTDs relative to the explicit FDTD somewhat depends on the model to be treated, such as a waveguide structure and a computational window size. For example, in the facet reflectivity analysis [20], the LOD-FDTD becomes efficient for CFLN $>5$, which is slightly different from that discussed just above.

In addition, the reduction of the CPU time with the LODFDTD reported in [20] (20\% reduction) is slightly larger than $10 \%-15 \%$ shown above. This difference is mainly due to the absorbing boundary condition (ABC) to be used. In [20], we applied a simple $A B C$ with a cosine function, which is often employed in optical waveguide analyses, to the ADIand LOD-FDTDs. The simple ABC is less effective than the PML but is enough for the case of the facet reflectivity analysis [20]. However, we have to use the PML in order to track the accurate TD responses with low amplitude and high frequency components for the grating analysis performed here. Notice that the PML requires more memory compared with the simple $\mathrm{ABC}$, giving rise to a long $\mathrm{CPU}$ time. Unfortunately, this becomes an overhead for both ADI- and LOD-FDTD calculations, resulting in the small difference between the CPU times of these two methods.

The computational efficiency is also checked in terms of the memory requirement. For the explicit FDTD, the implicit FDTD, and the envelope FDTD, the requirements are about 3, 7.5 , and $14 \mathrm{MB}$, respectively. The requirement of the explicit FDTD in this analysis is reduced to $\simeq 80 \%$ of that in [7]. This is because the unsplit-type PML used here requires less memory, in comparison with that of the split-type PML used in [7]. The memory requirements for the implicit and envelope FDTDs,

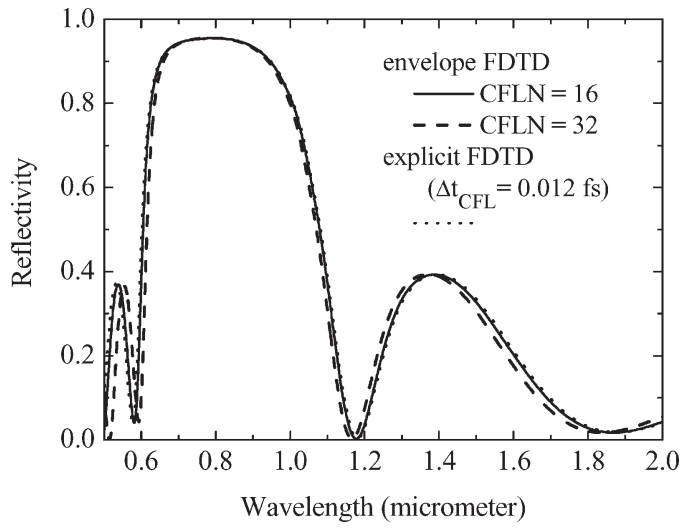

Fig. 5. Spectral response of the reflectivity for a waveguide with highreflection coatings [5].

which do not depend on the choice of $\Delta t$, are increased due to the solution of the tridiagonal matrix, in comparison with the explicit FDTD. The memory of the envelope FDTD is approximately twice that of the implicit FDTD, because of the complex envelope formulation.

As discussed above, although the envelope FDTD gives comparable results to those of the explicit FDTD, its advantage is not so clear. We finally investigate the case for a large CFLN with the envelope FDTD, in which the reduced CPU time is expected when compared to the explicit FDTD. We here analyze an optical waveguide with high-reflection coatings treated in [5] using the envelope FDTD. For this model, the small spatial sampling widths are required to obtain desired the accuracy: $\Delta x=0.0125 \mu \mathrm{m}$ and $\Delta z=0.004 \mu \mathrm{m}$ are used [5], resulting in $\Delta t_{\mathrm{CFL}}=0.012 \mathrm{fs}$ for the explicit FDTD. For the envelope FDTD, the time steps with CFLN $=16$ ( $\Delta t=0.2 \mathrm{fs}$ ) and $32(\Delta t=0.4 \mathrm{fs})$ are adopted, and $\lambda=0.8 \mu \mathrm{m}$ is used as a center wavelength.

Fig. 5 shows the spectral response of the reflectivity. It can be found that the results from the envelope FDTD are in good agreement with the result from the explicit FDTD, even for a large CFLN. These results attribute to a relatively low dispersion error found in Fig. 1(c). In this case, the CPU times, for instance, of the envelope LOD-FDTD are reduced to 50\% and $25 \%$ for CFLN $=16$ and 32, respectively, of the CPU time of the explicit FDTD. It is therefore expected that for problems requiring small spatial sampling widths, the envelope FDTD efficiently provides the numerical results when compared with the explicit FDTD.

\section{CONCLUSiON}

We have compared several FDTDs for the optical waveguide analyses. In particular, we develop efficient implicit FDTDs based on the LOD scheme. In the numerical dispersion analysis, the normalized phase velocity of the ADI/LOD-FDTD gradually degrades with an increase in the time step, while the velocities of the envelope versions remain close to unity at the center wavelength even for a large time step. As expected from these results, the envelope ADI/LOD-FDTD yields reasonably accurate spectral responses of the waveguide grating around the center wavelength. The CPU times of the LOD-based 
FDTDs are found to be reduced by $10 \%-15 \%$, in comparison with the ADI-based FDTDs. It is shown that the efficiency of the envelope LOD-FDTD becomes significant for CFLN $>8$ when compared with the explicit FDTD. Further calculations of a waveguide with high-reflection coatings reveal that the CPU time of the envelope LOD-FDTD with CFLN $=32$ is reduced to $25 \%$ of that of the explicit FDTD. The implicit FDTDs treated here may be extended to a full-vectorial threedimensional problem, which will be left for future study.

\section{ACKNOWLEDGMENT}

The authors would like to thank Dr. Y. Y. Lu of City University of Hong Kong for the invaluable discussions regarding TD methods.

\section{REFERENCES}

[1] K. S. Yee, "Numerical solution of initial boundary value problems involving Maxwell's equations in isotropic media," IEEE Trans. Antennas Propag., vol. AP-14, no. 3, pp. 302-307, May 1966.

[2] A. Taflove and S. C. Hagness, Computational Electrodynamics: The Finite-Difference Time-Domain Method. Norwood, MA: Artech House, 2000.

[3] S. T. Chu, W. P. Huang, and S. K. Chaudhuri, "Simulation and analysis of waveguide based optical integrated circuits," Comput. Phys. Commun., vol. 68, no. 1-3, pp. 451-484, Nov. 1991.

[4] J. Yamauchi, M. Mita, S. Aoki, and H. Nakano, "Analysis of antireflection coatings using the FD-TD method with the PML absorbing boundary condition," IEEE Photon. Technol. Lett., vol. 8, no. 2, pp. 239-241, Feb. 1996.

[5] J. Yamauchi, S. Sasaki, and H. Nakano, "Pulse excitation scheme for the analysis of wide-band characteristics of optical waveguides," IEEE Photon. Technol. Lett., vol. 17, no. 2, pp. 357-359, Feb. 2005.

[6] P. L. Liu, Q. Zhao, and F. S. Choa, "Slow-wave finite-difference beam propagation method," IEEE Photon. Technol. Lett., vol. 7, no. 8, pp. 890-892, Aug. 1995.

[7] J. Shibayama, M. Muraki, J. Yamauchi, and H. Nakano, "Comparative study of several time-domain methods for optical waveguide analyses," $J$. Lightw. Technol., vol. 23, no. 7, pp. 2285-2293, Jul. 2005.

[8] T. Namiki, "A new FDTD algorithm based on alternating-direction implicit method," IEEE Trans. Microw. Theory Tech., vol. 47, no. 10, pp. 2003-2007, Oct. 1999.

[9] F. H. Zheng, Z. Z. Chen, and J. Z. Zhang, "A finite-difference time-domain method without the Courant stability conditions," IEEE Microw. Guided Wave Lett., vol. 9, no. 11, pp. 441-443, Nov. 1999.

[10] F. Zhen, Z. Z. Chen, and J. Z. Zhang, "Toward the development of a three-dimensional unconditionally stable finite-difference timedomain method," IEEE Trans. Microw. Theory Tech., vol. 48, no. 9, pp. 1550-1558, Sep. 2000.

[11] T. Namiki, "3-D ADI-FDTD method-unconditionally stable time-domain algorithm for solving full vector Maxwell's equations," IEEE Trans. Microw. Theory Tech., vol. 48, no. 10, pp. 1743-1748, Oct. 2000.

[12] D. W. Peaceman and H. H. Rachford, Jr., "The numerical solution of parabolic and elliptic differential equations," J. Soc. Ind. Appl. Math., vol. 3, no. 1, pp. 28-41, Mar. 1955.

[13] A. R. Mitchell and D. F. Griffiths, The Finite-Difference Method in Partial Differential Equations. Hoboken, NJ: Wiley, 1980.

[14] S. G. Garcia, T. W. Lee, and S. C. Hagness, "On the accuracy of the ADI-FDTD method," IEEE Antennas Wireless Propag. Lett., vol. 1, no. 1, pp. 31-34, 2002.

[15] A. P. Zhao, "Analysis of the numerical dispersion of the 2D alternatingdirection implicit FDTD method," IEEE Trans. Microw. Theory Tech., vol. 50, no. 4, pp. 1156-1164, Apr. 2002.

[16] G. Sun and C. W. Trueman, "Analysis and numerical experiments on the numerical dispersion of two-dimensional ADI-FDTD," IEEE Antennas Wireless Propag. Lett., vol. 2, no. 1, pp. 78-81, 2003.

[17] S. Ju, H. Kim, and H. H. Kim, "A study of the numerical dispersion relation for the 2-D ADI-FDTD method," IEEE Microw. Wireless Compon. Lett., vol. 13, no. 9, pp. 405-407, Sep. 2003.

[18] G. L. Sun and C. W. Trueman, "Some fundamental characteristics of the one-dimensional alternate-direction-implicit finite-difference time- domain method," IEEE Trans. Microw. Theory Tech., vol. 52, pt. 1, no. 1, pp. 46-52, Jan. 2004.

[19] H. Rao, R. Scarmozzino, and R. M. Osgood, Jr., "An improved ADIFDTD method and its application to photonic simulations," IEEE Photon. Technol. Lett., vol. 14, no. 4, pp. 477-479, Apr. 2002.

[20] J. Shibayama, M. Muraki, J. Yamauchi, and H. Nakano, "Efficient implicit FDTD algorithm based on locally one-dimensional scheme," Electron. Lett., vol. 41, no. 19, pp. 1046-1047, Sep. 2005. See also Errata, Electron. Lett., vol. 42, no. 8, p. 497, Apr. 2006

[21] S. Ju, K. Y. Jung, and H. Kim, "Investigation on the characteristics of the envelope FDTD based on the alternating direction implicit scheme," IEEE Microw. Wireless Compon. Lett., vol. 13, no. 9, pp. 414-416, Sep. 2003.

[22] C. N. Ma and Z. Z. Chen, "Dispersion analysis of the three-dimensional complex envelope ADI-FDTD method," IEEE Trans. Antennas Propag., vol. 53, no. 3, pp. 971-976, Mar. 2005.

[23] M. Wang, Z. Wang, and J. Chen, "A parameter optimized ADI-FDTD method," IEEE Antennas Wireless Propag. Lett., vol. 2, no. 1, pp. 118 121, 2003.

[24] A. P. Zhao, "Improvement on the numerical dispersion of 2-D ADI-FDTD with artificial anisotropy," IEEE Microw. Wireless Compon. Lett., vol. 14, no. 6, pp. 292-294, Jun. 2004.

[25] H.-X. Zheng and K. W. Leung, "An efficient method to reduce the numerical dispersion in the ADI-FDTD," IEEE Trans. Microw. Theory Tech., vol. 53, no. 7, pp. 2295-2301, Jul. 2005.

[26] J. Chen, Z. Wang, and Y. Chen, "Higher-order alternative direction implicit FDTD method," Electron. Lett., vol. 38, no. 22, pp. 1321-1322, Oct. 2002.

[27] Z. Wang, J. Chen, and Y. C. Chen, "Development of a higher-order ADI-FDTD method," Microw. Opt. Technol. Lett., vol. 37, no. 1, pp. 8-12, Apr. 2003.

[28] Y. Y. Lu, "New unconditionally stable ADI schemes for the wave equation," presented at the CLEO/Pacific Rim, Taipei, Taiwan, R.O.C., 2003, Paper W1A-(14)-2.

[29] W. Fu and E. L. Tan, "Development of split-step FDTD method with higher-order spatial accuracy," Electron. Lett., vol. 40, no. 20, pp. 1252-1253, Sep. 2004.

[30] W. M. Fu and E. L. Tan, "A compact higher-order ADI-FDTD method," Microw. Opt. Technol. Lett., vol. 44, no. 3, pp. 273-275, Feb. 2005.

[31] M. K. Sun and W. Y. Tam, "Stability and dispersion analysis of ADIMRTD and ADI high-order schemes," Microw. Opt. Technol. Lett., vol. 45, no. 1, pp. 43-46, Apr. 2005.

[32] S. Wang, F. L. Teixeira, and J. Chen, "An iterative ADI-FDTD with reduced splitting error," IEEE Microw. Wireless Compon. Lett., vol. 15, no. 2, pp. 92-94, Jul. 2005.

[33] B. Donderici and F. L. Teixeira, "Symmetric source implementation for the ADI-FDTD method," IEEE Trans. Antennas Propag., vol. 53, no. 4, pp. $1562-1565$, Apr. 2005.

[34] S. M. Wang, "On the current source implementation for the ADIFDTD method," IEEE Microw. Wireless Compon. Lett., vol. 14, no. 11, pp. 513-515, Nov. 2004.

[35] S. D. Gedney, G. Liu, J. A. Roden, and A. Zhu, "Perfectly matched layer media with CFS for an unconditionally stable ADI-FDTD method," IEEE Trans. Antennas Propag., vol. 49, no. 11, pp. 1554-1559, Nov. 2001.

[36] S. Wang and F. L. Teixeira, "An efficient PML implementation for the ADI-FDTD method," IEEE Microw. Wireless Compon. Lett., vol. 13, no. 2, pp. 72-74, Feb. 2003.

[37] O. Ramadan, "Unconditionally stable nearly PML algorithm for linear dispersive media," IEEE Microw. Wireless Compon. Lett., vol. 15, no. 7, pp. 490-492, Jul. 2005.

[38] J. P. Berenger, "Numerical reflection from FDTD-PMLs: A comparison of the split PML with the unsplit and CFS PMLs," IEEE Trans. Antennas Propag., vol. 50, no. 3, pp. 258-265, Mar. 2002.

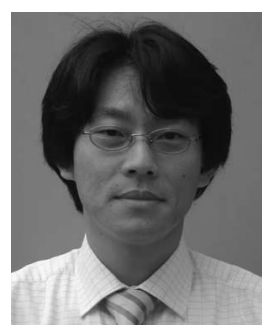

Jun Shibayama (M'03) was born in Kashiwa, Japan, on July 1, 1969. He received the B.E., M.E., and Dr. E. degrees from Hosei University, Tokyo, Japan, in 1993, 1995, and 2001, respectively.

In 1995, he joined Opto-Technology Laboratory (currently FITEL Photonics Laboratory), Furukawa Electric Company, Ltd., Ichihara, Chiba, Japan. Since 1999, he has been an Assistant with Hosei University. His research interest includes numerical analysis of optical waveguides.

Dr. Shibayama is a member of the Optical Society of America (OSA) and the Institute of Electronics, Information, and Communication Engineers (IEICE) of Japan. 


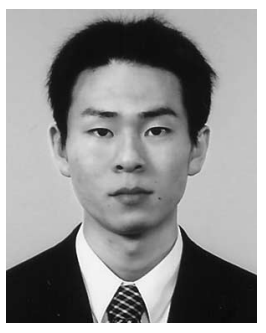

Mitsunori Muraki was born in Saitama, Japan, on April 5, 1981. He received the B.E. degree from Hosei University, Tokyo, Japan, in 2004, where he is currently working toward the M.E. degree.

Mr. Muraki is a student member of the Institute of Electronics, Information, and Communication Engineers (IEICE) of Japan.

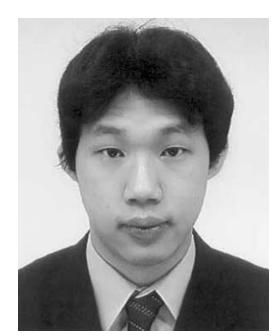

Ryo Takahashi was born in Niigata, Japan, on August 31,1983 . He is currently working toward the B.E. degree at Hosei University, Tokyo, Japan.

Mr. Takahashi is a student member of the Institute of Electronics, Information, and Communication Engineers (IEICE) of Japan.

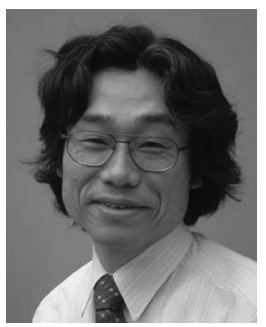

Junji Yamauchi (M'84) was born in Nagoya, Japan, on August 23, 1953. He received the B.E., M.E., and Dr. E. degrees from Hosei University, Tokyo, Japan, in 1976, 1978, and 1982, respectively.

From 1984 to 1988, he served as a Lecturer in the Electrical Engineering Department of Tokyo Metropolitan Technical College. Since 1988, he has been a member of the faculty of Hosei University, where he is now a Professor of electronic informatics. His research interests include optical waveguides and circularly polarized antennas. He is the author of Propagating Beam Analysis of Optical Waveguides (Baldock, Hertfordshire, U.K.: Research Studies, 2003).

Dr. Yamauchi is a member of the Optical Society of America (OSA) and the Institute of Electronics, Information, and Communication Engineers (IEICE) of Japan.

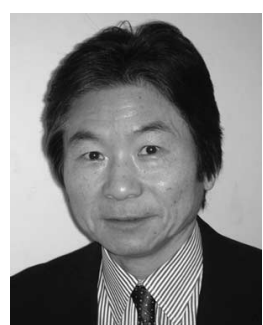

Hisamatsu Nakano (M'75-SM'87-F'92) was born in Ibaraki, Japan, on April 13, 1945. He received the B.E., M.E., and Dr. E. degrees in electrical engineering from Hosei University, Tokyo, Japan, in 1968, 1970, and 1974, respectively.

Since 1973, he has been a member of the faculty of Hosei University, where he is currently a Professor of electronic informatics. His research topics include numerical methods for low- and high-frequency antennas and optical waveguides. He has published more than 200 refereed journal papers, more than 200 international-symposium papers, and more than 750 national-symposium papers. He is the author of a book entitled Helical and Spiral Antennas (New York: Research Studies, Wiley, 1987) and the coauthor of Analysis Methods of Electromagnetic Wave Problems, Volume Two (Norwood, MA: Artech House, 1986). In addition, he is the author of Helical and Spiral Antennas, Encyclopedia of Communications (New York: Wiley, 2002).

Dr. Nakano received the IEE International Conference on Antennas and Propagation Best Paper Award and the IEEE TRANSACTIONS ON ANTENNAS AND Propagation Best Application Paper Award (H. A. Wheeler Award) in 1989 and 1994, respectively. In 1992, he was elected an IEEE Fellow for the contributions to the design of spiral and helical antennas. In 2001, he received the Award of Distinguished Technical Communication from the Society for Technical Communication, USA, and the Science and Technology Progress Award, from Hangzhou, China. He is an Associate Editor of several journals and magazines, such as Electromagnetics, IEEE ANTENNAS AND PRopagation MAgazine, IEEE ANTENNAS AND Wireless PROPAGATION LETTERS, and ASIAN INFORMATION-SCIENCE-LIFE. 